\title{
Human Papillomavirus Vaccine
}

\author{
Yu Jeung Lee \\ Drug Information Research Institute, Sookmyung Women's University, Seoul, Korea
}

(Received 8 June 2007; Accepted 18 July 2007)

\begin{abstract}
Human papillomavirus (HPV) infection is the most common sexually transmitted disease in the United States. An estimated 6.2 million people are infected with HPV every year. Randomized controlled studies consistently show that HPV vaccine is effective in preventing infection and HPV related cervical lesions. In June 2006, Gardasil (qadrivalent HPV recombinant vaccine) was approved by the FDA for use in females 9-26 years of age. This article reviews published data to evaluate the effectiveness of HPV vaccine for the prevention of cervical cancer.
\end{abstract}

Key words $\square$ cervical cancer, human papillomavirus vaccine, Gardasil

\section{INTRODUCTION}

Human papillomavirus (HPV) infection is the most common sexually transmitted disease today (Trottier and Franco, 2006). The Centers for Disease Control and Prevention estimates that approximately 20 million Americans are currently infected with HPV. About 6.2 million Americans become infected with genital HPV each year and that more than half of all sexually active men and women become infected at some time in their lives (Centers for Disease Control and Prevention, 2007).

Molecular epidemiologic evidence clearly indicates that certain types of HPV are the principal cause of invasive cervical cancer and cervical intraepithelial neoplasia (Munoz et al., 2003). More than 80 types of HPV have been identified, and about 40 can infect the epithelial lining of the anogenital tract and other mucosal areas of the body (Trottier and Franco, 2006; Munoz et al., 2003). Cervical cancer is the second most common cancer among women worldwide, with an estimated 493,000 new cases and 274,000 deaths in 2002. The highest incidence rates are observed in sub-Saharan Africa, Melanesia, Latin America and the Caribbean, South-Central Asia, and South East Asia (Parkin and Bray, 2006).

The US Food and Drug Administration (FDA) announced on June 8, 2006, the approval of Gardasil (qadrivalent HPV recombinant vaccine), the first vaccine developed to prevent

*Corresponding author

Tel: +82-2-710-9264, Fax: +82-2-702-5728

E-mail: yjlee@sdic.sookmyung.ac.kr cervical cancer, precancerous genital lesions, and genital warts caused by HPV types 6, 11, 16, and 18 (FDA News, 2007). The vaccine is approved for use in females 9-26 years of age (FDA News, 2007; Gardasil prescribing information, 2006). In May 2007, Cervarix (bivalent HPV recombinant vaccine) got an approval in Australia for females 10-45 years old. The objective of this article is to evaluate the available data regarding the effectiveness of HPV vaccine for the prevention of cervical cancer.

\section{HUMAN PAPILLOMAVIRUS}

HPV is a small DNA tumor virus that has a circular genome of approximately 8,000 base-pairs and an icosohedral viral capsid composed of two proteins, L1 and L2 (Wright et al., 2006). The genome has the coding capacity for these two proteins and at least six so-called early proteins (E1, E2, E4-E7) that are necessary for the replication of the viral DNA and for the assembly of newly produced virus particles within the infected cells (Munoz et al., 2006). The genital HPV types can be divided into three groups: "high-risk" or "carcinogenic" types $(16,18,31,33,35,39,45,51,52,56,58,59)$ associated with a high relative risk of cervical cancer; "low risk" types associated with benign epithelial proliferations in the genital area, but not associated with invasive cervical cancer $(6,11,40$, $42,43,44,54,61,70,72,81,89)$; and a group of six types (26, $53,66,68,73,82)$ that is classified as "probably carcinogenic" since there is limited data associating these HPV types with cervical cancer (Wright et al., 2006). HPV types 16 and 18 
cause approximately 70\% of cervical cancers, and HPV types 6 and 11 cause approximately $90 \%$ of genital warts.

The immune response that HPV triggers in humans is poor compared to that elicited by other viruses (Frazer, 1996). HPV is perfectly adapted to its natural host tissue, the differentiating epithelial cell of skin or mucosa, and exploits the cellular machinery for its own purposes. The cycle is initiated when infectious particles reach the basal layer of the epithelium, where they bind to and enter into cells, through small breaks. It has been suggested that for maintenance of the infection, the virus has to infect an epithelial stem cell (Munoz et al., 2006). As the HPV progresses through the layers of the epithelium, the replicative program of its genes changes in an orderly fashion. The early proteins E6 and E7, elemental to the transformation process, also cause epithelial cells to abort apoptosis. These early proteins are produced through most of the phases of the HPV life cycle, making them better candidates for therapeutic vaccines. On the other hand, the HPV L1 and L2 late proteins are not produced until the virus is located in the most superficial layers of the epithelium, correlating with the assembly of infectious virions and their release from the epithelium with the desquamated infected superficial cells (Padilla-Paz L, 2005).

\section{LITERATURE REVIEW}

Koutsky LA, Ault KA, Wheeler CM, et al conducted a double-blind, multicenter, randomized clinical study to determine whether a HPV 16 L1 virus-like-particle vaccine could prevent HPV 16 infection in women (Koutsky et al, 2002). This study enrolled 2,392 young women, defined as female subjects 16 to 23 years of age, who were not pregnant, reported no prior abnormal Pap smears, and reported that they had had no more than five male sex partners during their lifetime. They were randomly assigned to receive three intramuscular injections of either HPV 16 vaccine or placebo at day 0 , month 2 , and month 6. Genital samples to test for HPV 16 DNA were obtained at enrollment, one month after the third vaccination, and every six months thereafter. Follow-up visits were scheduled one month after the third vaccination (month 7), six months after the third vaccination (month 12), and every six months thereafter until month 48 . The primary endpoint was persistent HPV 16 infection, defined as the detection of HPV 16 DNA in samples obtained at two or more visits. Women were excluded from analysis of the primary endpoint if they were positive for HPV 16 infection at day 0 or month 7. A total of 1,533 women were included in the primary analysis. Of the 1,533 women, 768 women received vaccine and 765 women received placebo. Participants were followed for a median of 17.4 months after completing the vaccination regimen. The incidence of persistent HPV 16 infection was 3.8 per 100 woman-years at risk in the placebo group and 0 per 100 woman-years at risk in the vaccine group (100\% efficacy; 95\% confidence interval, 90 to $100 ; \mathrm{P}<0.001)$. Forty-one primary endpoint events were detected after month 7 of the study. Thirty-one were persistent HPV 16 infections without cervical intraepithelial neoplasia, 5 consisted of HPV 16 related cervical intraepithelial neoplasia grade 1, 4 consisted of HPV 16 related cervical intraepithelial neoplasia grade 2, and 1 occurred in a woman who was HPV 16 DNA positive for the first time on the last visit before she was lost to follow-up. All of these events occurred in the placebo group, and there were no events in the vaccine group.

A double blind, multicenter, randomized, placebo-controlled clinical study assessed the efficacy of a bivalent HPV 16/18 virus-like particle vaccine against HPV 16 and HPV 18 (Harper et al, 2004). This study included 1,113 healthy women between the ages of 15 and 25 years. Women were accepted only if they had had no more than six sexual partners, no history of an abnormal Pap smears or ablative or excisional treatment of the cervix, no ongoing treatment for external genital warts, and if they were seronegative for HPV 16 and 18 and DNA-negative for high-risk HPV types within 90 days prior to enrollment. The researchers randomized 560 women to receive bivalent HPV 16, 18 L1 virus-like-particle vaccine and 553 women to receive placebo at day 0 , month 1 , and month 6 . They did both according-to-protocol and intention-to-treat analyses for efficacy. In the according-to-protocol analysis, 366 women in the vaccine group and 355 women in the placebo group were included. The major reasons for elimination from according-toprotocol analysis were abnormal cytology, high-risk HPV DNA positivity, or seropositivity for HPV 16 or HPV 18 at enrollment. The primary endpoint was prevention of incident HPV 16 and 18 infection. In the according-to-protocol, vaccine efficacy against incident HPV 18 infection was not statistically significant $(72.3 \%$, 95\% confidence interval, -32.5 to 93.4 ; $\mathrm{P}=0.102)$. However, in the intention-to-treat cohort, vaccine efficacy was significant $(82.1 \%, 95 \%$ confidence interval, 38.8 to $94.7 ; \mathrm{P}=0.002$ ). This study showed $100 \%$ vaccine efficacy in the according-to-protocol against persistent HPV $16(100 \%$, $95 \%$ confidence interval, 47.0 to $100 ; \mathrm{P}=0.007$ in cervical samples) and HPV 16/18 infections (100\%, 95\% confidence interval, 47.0 to $100 ; \mathrm{P}=0.007$ in cervical samples).

In 2005 , another study was conducted to assess the efficacy 
of a prophylactic quadrivalent vaccine targeting HPV types 6 , 11, 16 and 18. This study was a phase II randomized, multicenter, double-blind, placebo-controlled study (Villa et al, 2005). In this study, 277 women aged 16-23 years were assigned to receive quadrivalent HPV L1 virus-like-particle vaccine and 275 women were assigned to receive placebo. Healthy women were enrolled if they were not pregnant, had no previous abnormal Pap smears, and reported a lifetime history of four or fewer male sex partners. This study did not exclude women with previous HPV infection. The primary endpoint was a persistent infection associated with HPV 6, 11, 16 , or 18 , or cervical or external genital disease compared with placebo. Both according-to-protocol and modified intention-totreat analyses for efficacy were done in this study. Over the 30 months' follow-up after vaccination, combined incidence of persistent HPV 6, 11, 16, or 18 infection or associated genital disease decreased by $90 \%$ (95\% confidence interval, 71 to 97 ; $\mathrm{P}<0.0001$ ) in women assigned vaccine compared with those assigned placebo. In the modified intention-to-treat cohort, the efficacy of the vaccine with regard to the primary endpoint was $89 \%$ (95\% confidence interval, 73 to 96 ; $\mathrm{P}<0.0001$ ).

The FUTURE II Study Group conducted a phase III trial to evaluate a quadrivalent vaccine against HPV types 6, 11, 16, and 18 (FUTURE II Study Group, 2007). This study was a multicenter, double-blind, randomized, placebo-controlled study enrolled 12,167 women aged 16-23 years. Women were eligible to participate in the study if they were not pregnant, did not report abnormal results on a Pap smear, and had had a lifetime number of no more than four sex partners. Participants were randomly assigned to receive vaccine or placebo at day 1 , month 2, and month 6 after having a negative result on a pregnancy test. Of a total of 12,167 women, 6,087 were assigned to receive vaccine and 6,080 to receive placebo. They were followed for an average of 3 years after receiving the first dose of vaccine or placebo. The primary endpoint was cervical intraepithelial neoplasia grade 2 or 3 , adenocarcinoma in situ, or invasive carcinoma of the cervix, with the detection of DNA from HPV 16, HPV 18, or both in one or more of three adjacent sections of the same lesion. The researchers carried out both according-to-protocol and intention-to-treat analyses. The vaccine prevented $98 \%$ of HPV 16/18 related high-grade cervical lesions $(95.89 \%$ confidence interval, 86 to $100 ; \mathrm{P}<0.001)$ in the according-to-protocol susceptible population. In the vaccine group, there was 1 case of HPV 16/18 related high-grade cervical lesions compared with 42 cases in the placebo group. The vaccine prevented $44 \%$ (95\% confidence interval, 26 to 58 ) in the intention-to-treat population. In the vaccine group, there were 83 cases of HPV 16/18 related high-grade cervical lesions compared with 148 cases in the placebo group. The estimated vaccine efficacy against all high-grade cervical lesions associated with any HPV type was $17 \%$ (95\% confidence interval, 1 to 31$)$ in the intention-to-treat population.

\section{SUMMARY}

The results from four randomized controlled studies demonstrate that HPV vaccine is effective in preventing HPV infection and HPV related cervical lesions (Koutsky et al., 2002; Harper et al., 2004 ; Villa et al., 2005; FUTURE II Study Group, 2007). However, there are limitations in the above studies. HPV vaccine was developed relatively recently. Therefore, information about durability of HPV vaccine induced protection is limited. HPV vaccine may be used for prevention of genital warts in men, but all of studies included only women in the patient sample.

In the developing nations, HPV 16 and 18 account for about 64-74\% of cervical cancer (Muñoz et al., 2004). Advisory Committee for Immunization Practices (ACIP) recommended that Gardasil (qadrivalent HPV recombinant vaccine) be routinely administered to girls when they are 11 to 12 years old. However, we should determine the optimal age for vaccination before HPV vaccine is introduced in Korea since sexual culture is different between Korea and the United States.

\section{REFERENCES}

Centers for Disease Control and Prevention. (2007). Genital HPV Infection - CDC Fact Sheet.

FDA News. (2007) FDA Licenses New Vaccine for Prevention of Cervical Cancer and Other Diseases in Females Caused by Human Papillomavirus.

Frazer I. H. (1996). Immunology of papillomavirus infection. Curr. Opin. Immunol. 8(4), 484-91.

FUTURE II Study Group. (2007). Quadrivalent vaccine against human papillomavirus to prevent high-grade cervical lesions. N. Engl. J. Med. 356(19), 1991-3.

Gardasil [Quadrivalent Human Papillomavirus (Types 6, 11, 16, 18) Recombinant Vaccine] prescribing information. (2006). Whitehouse Station, N.J.

Harper D. M., Franco E. L., Wheeler C., Ferris D. G., Jenkins D., Schuind A., Zahaf T., Innis B., Naud P., De Carvalho N. S., Roteli-Martins C. M., Teixeira J., Blatter M. M., Korn A. P., Quint W. and Dubin G. (2004). Efficacy of a bivalent L1 viruslike particle vaccine in prevention of infection with human papillomavirus types 16 and 18 in young women: a randomised controlled trial. Lancet 364(9447), 1757-65.

Koutsky L. A., Ault K. A., Wheeler C.M., Brown D. R., Barr E., Alvarez F. B., Chiacchierini L. M. and Jansen K. U. (2002). A 
controlled trial of a human papillomavirus type 16 vaccine. $N$. Engl. J. Med. 347(21), 1645-51.

Muñoz N, Bosch F. X., Castellsagué X., Díaz M., de Sanjose S., Hammouda D., Shah K. V. and Meijer C. J. (2004). Against which human papillomavirus types shall we vaccinate and screen? The international perspective. Int. J. Cancer. 111(2), $278-85$.

Munoz N., Bosch F. X., de Sanjose S., Herrero R., Castellsagué X., Shah K. V., Snijders P. J. and Meijer C. J. (2003). Epidemiologic classification of human papillomavirus types associated with cervical cancer. N. Engl. J. Med. 348(6), 518-27.

Munoz N., Castellsague X., de Gonzalez A. B. and Gissmann L. (2006). Chapter 1: HPV in the etiology of human cancer. Vaccine 24(S3), S1-10.

Padilla-Paz L. A. (2005). Human papillomavirus vaccine: history, immunology, current status, and future prospects. Clin. Obstet. Gynecol. 48(1), 226-40.

Parkin D. M. and Bray F. (2006) Chapter 2: The burden of HPVrelated cancers. Vaccine 24(S3), S11-25.
Trottier H. and Franco E. L. (2006). The epidemiology of genital human papillomavirus infection. Vaccine 24(S1), S4-15.

Villa L. L., Costa R. L., Petta C. A., Andrade R. P., Ault K. A., Giuliano A. R., Wheeler C. M., Koutsky L. A., Malm C., Lehtinen M., Skjeldestad F. E., Olsson S. E., Steinwall M., Brown D. R., Kurman R. J., Ronnett B. M., Stoler M. H., Ferenczy A., Harper D. M., Tamms G. M., Yu J., Lupinacci L., Railkar R., Taddeo F. J., Jansen K. U., Esser M. T., Sings H. L., Saah A. J. and Barr E. (2005). Prophylactic quadrivalent human papillomavirus (types 6, 11, 16, and 18) L1 virus-like particle vaccine in young women: a randomised double-blind placebo-controlled multicentre phase II efficacy trial. Lancet Oncol. 6(5), 271-8.

Wright T. C., Bosch F. X., Franco E. L., Cuzick J., Schiller J. T., Garnett G. P. and Meheus A. (2006). Chapter 30: HPV vaccines and screening in the prevention of cervical cancer; conclusions from a 2006 workshop of international experts. Vaccine 24(S3), S251-61. 